\title{
Medical Data Management Using Blockchain
}

\author{
Bhalaji N*, Aishwarya V, Krithika Balaji \\ Information Technology, Sri Sivasubramaniya Nadar College of Engineering, India \\ E-mail: *bhalajin@it.ssn.edu.in
}

\begin{abstract}
Medical data of a person is an extremely sensitive and valuable resource. If this data gets into the wrong hands, it can be misused in unimaginable ways. Any organization handling this sort of sensitive data is taking on an extremely huge responsibility. A patient should not have to worry about the safety of his data in the hands of a third-party organization. If he is given control of his sensitive data by eliminating the middle man, then handling such data would become a lot easier. This is where the concept of blockchain is introduced. The blockchain technology proves to be an excellent technology to safeguard such data. A blockchain application which connects multiple entities like hospitals, labs, patients and doctors proves to be the perfect solution to ensure that there is no tampering of data.
\end{abstract}

Keywords - Blockchain, Decentralization

\section{INTRODUCTION}

Healthcare is an aggregate and combination of divisions within the economic system that procures goods and services to handle patients with therapeutic, defensive, and rehabilitative concerns. This industry is devoted towards prevention, diagnosis and treatment of medical conditions. It involves large amounts of patient data that consists of sensitive information. It is important to ensure the protection of such information. This will help to safeguard data and to be used for the right purposes alone. To protect healthcare data, the blockchain is used.

This project focusses on protecting the security of data in the healthcare industry. The primary objective of protecting data is to protect the interests of individuals. The information gathered is stored securely for further usage. The data security is to have control over the access for those who require data.

The common processing managed by the hospital is to maintain the Electronic Health Record (EHR), where all the patient's details are stored and retrieved whenever necessary. Many applications are used by hospital authorities to monitor patient data.

As computers have become an important part of our lives, it is essential to consider the protection of data in our list of priorities. The risk factors faced by the healthcare sector are overcome through the digitization of information. Maintaining the confidentiality of the patient's is the backbone of medicine. Protection over the sensitive information of the patients is the relationship of trust between the individual and the doctor. Blockchain technology is an acceptable concept for the healthcare space, and this leads to accessing the information securely. Information needs to be shared between the hosts confidentially. There are numerous solutions available for EMR/EHR solutions, but those are represented in the form of their own languages. Due to this, patients information are distributed crosswise companies/locations, and these involve huge maintenance cost. After combining all together, the patients felt they were provided improper care or inappropriate care. To overcome the 
difficulty, the blockchain serves as the arbitrator for the whole universe to merge the data under a single framework. It also increases the flow of data between the systems with reduced costs. All the processing include as checkups, medication, issues, and so on, can be recorded under a single central ledger and the EMR system can access the patient history without any interruption.

All the major healthcare sectors around the world store their data within their organizations. This is a decent system except for the fact that the data is stored in a centralized database and access to this data is limited to certain authorities. A healthcare organization should not be given complete control of such sensitive information of individuals. Though they follow various norms of handling the data, a loophole will always be present and this sensitive data can be misused by various companies for a variety of reasons. This is one of the main reasons behind trying to implement the blockchain technology into handling the medical data.

EHR platform permits the healthcare providers to process the operations such as to edit, view, and share the patient's information. Some of the challenges faced by the health data are correlation between the patient's records, interchanged health information, and interoperability. The solutions that can be provided to these kinds of data are framing under electronic format.

The objective of the blockchain is to permit secure data over the medical records. It is responsible for providing authentication for the users who require those data. The single-chain management designates an individual patient's medical document. The data entry occurs within the chain and every single data can be viewed by the patient's care team. The National blockchain-based approach assists in the progress of the information along with the patients. Blockchain technology ensures that the data structure is append-only and therefore it cannot be altered or deleted. It makes the storage of data transparent so that individuals or organizations who are provided authority can view the data. The advantages that come along with the usage of blockchain technology is plenty.

Blockchain technology ensures that the data structure is append-only and therefore it cannot be altered or deleted. It makes the storage of data transparent so that individuals or organizations who are provided authority can view the data. The advantages that come along with the usage of blockchain technology is plenty.

The key deliverables of this project include an end-to-end application which will focus on the interaction between the three entities (patients, doctors and labs) and the formation of the blocks which eventually get included into a blockchain using the Hyperledger fabric.

\section{SYSTEM ARCHITECTURE}

A. System Design

The architecture of the system has mainly three layers - Client Tier, Business Tier and Database Tier as shown in the figure below:

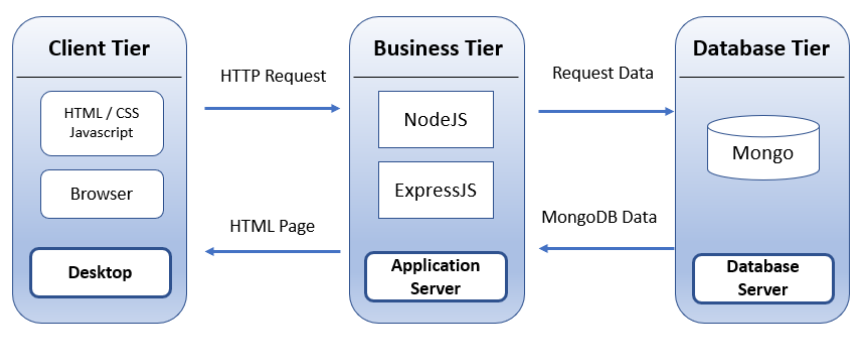

Fig. 1 System architecture with multiple layers 
The above-mentioned layers are commonly called the three-tier architecture of a web application.

B. Presentation layer

The presentation layer represents the graphical user interface(GUI), which serves in the interaction between the data and application layer. This layer is developed using HTML5, Javascript, Bootstrap 4, and Cascading Style Sheets (CSS) to employ the devices within a web-based application. It also interacts with other layers by the application program interface (API) requests. This layer will not include the data code and business logic.

\section{Application layer}

The application layer or logic layer was formulated in the server-side scripting language called Nodejs. It comprises the application's central functionalities which are termes as business logic. The process is entertained toward a dedicated in-house server. It should, later on, be hosted on the cloud to increase the processing power as in turn the scalability of the application. This layer contains the set of rules for processing the application. It can also accommodate many users.

D. Data layer

The data layer handles the writing and reading operations to access a database. This layer is also referred to as the storage layer. It can later be hosted in the cloud to increase scalability. MongoDB is the database used in this application. It is classified as a NoSQL database program, and it uses JSONlike documents with the schema.

\section{TOOLS AND ENVIRONMENT}

\section{A. Hyperledger Fabric}

Hyperledger Fabric works like a framework for application development within a modular architecture. Various components include plug-and-play, consensus, and membership services are basic to classify the different use cases in the industry. The consensus component facilitates performance during privacy preservation. It is a ledger platform and the enterprise-grade to produce versatility and modularity for different use cases. IBM developed an industrial enterprise, that can be utilized as a Fabric for designing a highly-modular called Decentralized Ledger Technology (DLT). The functionality of Fabric 2.0 is updating smart contract technology, smooth data sharing, and more active transactions. It serves as an open-source engine for blockchain management and responsible for feature evaluation.

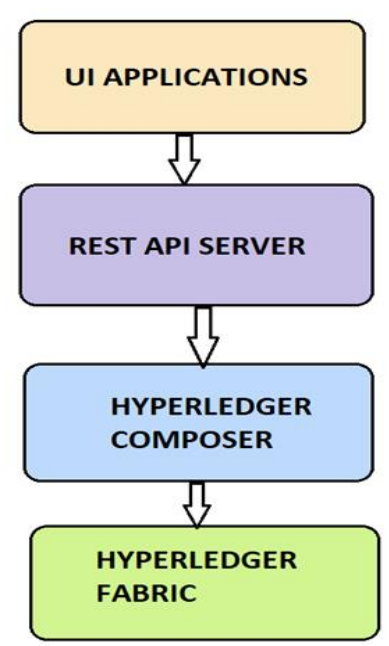


Fig. 24 layers of the system

a) UI Applications - They are web applications used to easily use the network

b) REST API Server - Exposes APIs for UI applications to interact with the network by using Composer SDK

c) Hyperledger Composer - Blockchain application development framework, simplified modelling language provides SDK for Node.js

d) Hyperledger Fabric - Blockchain runtime, consensus, ledger provides SDKs in Node.js

B. Docker

One of the familiar tools designed to deploy, run, and create an application termed Docker under the control of containers. It also uses containers to assist the features of the Linux kernel alike namespaces. And also assist to create control groups on the top of an operating system and helps to function the application deployment automatically using the containers. The application is developed using packages such as libraries and other provinces. Docker provides security when the application is executed over the common environment. There are three categories of containers available they are builder, engine, and orchestration. The builder is utilized as containers. The engine aids to execute the container. The orchestration supports to maintain the containers.

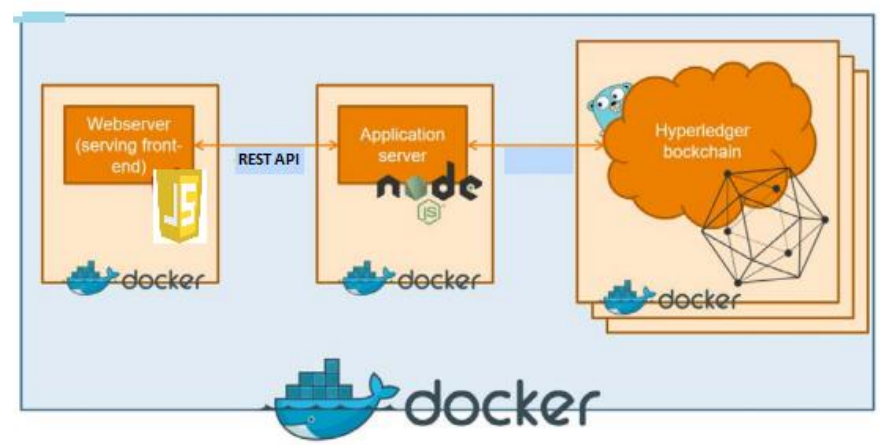

Fig. 3 Docker Container

C. Advanced REST Client

Advanced REST Client API is utilized to examine the communication between different levels of software components. The API endpoints validate the return values using the testing process. The responses can be recorded in terms of success false or success true which is tested by various testing techniques. In Advanced REST Client API requests are performed by GET and POST methods. The key values are transferred using the URL in the GET method and those values are hidden while transferring using the POST method. The GET and the POST methods are the two possible ways of transferring the information between the browser and server using the HTTP request. 
Journal of ISMAC (2020)

Vol.02/ No.04

Pages: 222-231

http://irojournals.com/iroismac/

DOI: https://doi.org/10.36548/jismac.2020.4.006

\section{OUTPUT OF PROJECT}

BlockCare

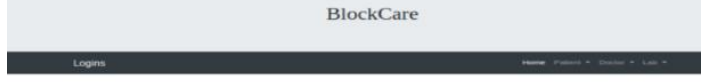

BlockCare

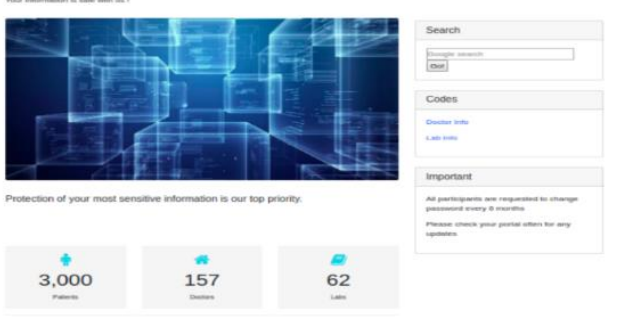

Fig. 4 Home Screen

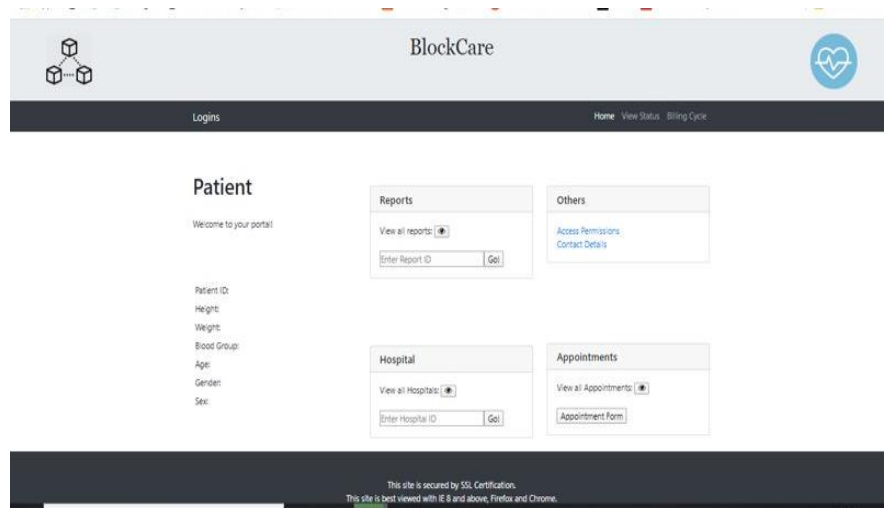

Fig. 5 Patient Dashboard

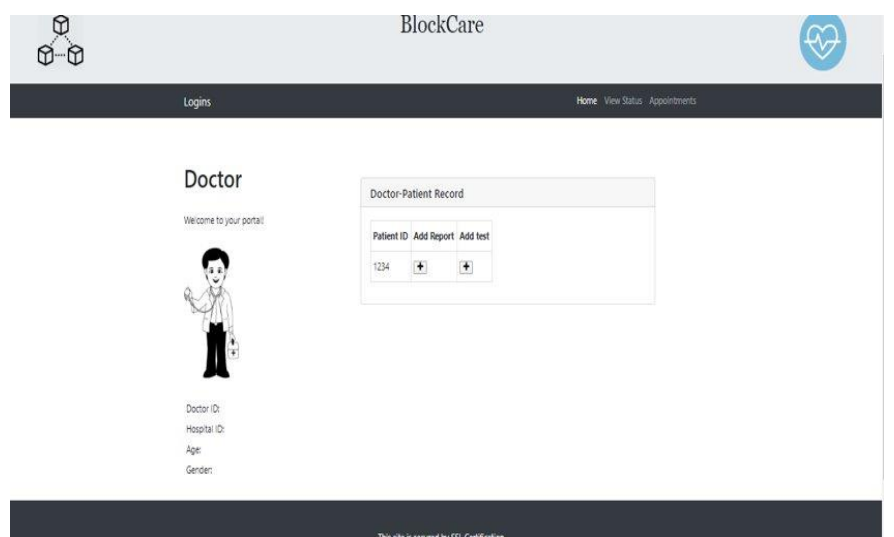

Fig. 6 Doctor Dashboard 
Journal of ISMAC (2020)

Vol.02/ No.04

Pages: 222-231

http://irojournals.com/iroismac/

DOI: https://doi.org/10.36548/jismac.2020.4.006

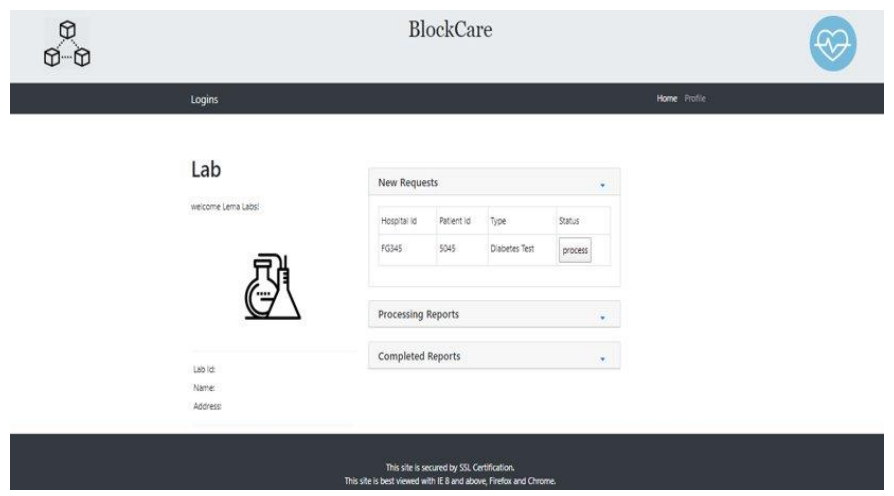

Fig. 7 Lab Dashboard

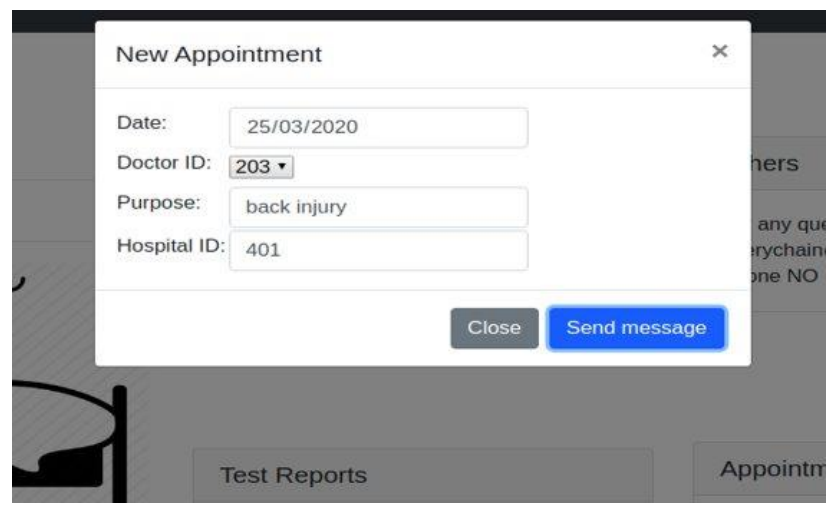

Fig. 8 Make Appointment

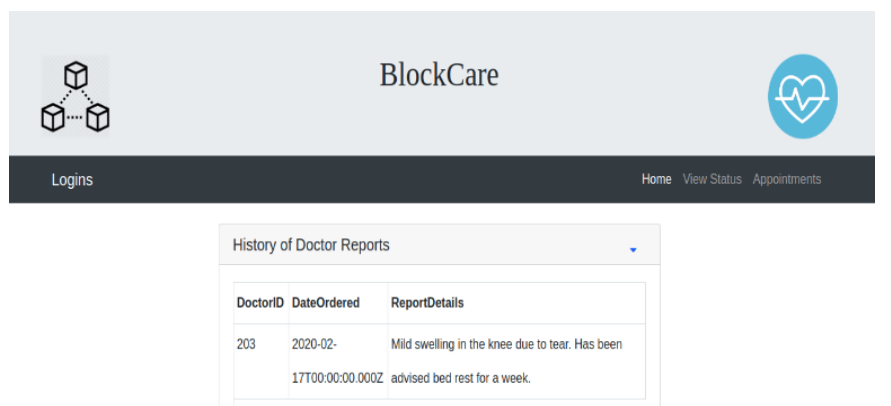

Fig. 9 Doctor Report 
Journal of ISMAC (2020)

Vol.02/ No.04

Pages: 222-231

http://irojournals.com/iroismac/

DOI: https://doi.org/10.36548/jismac.2020.4.006
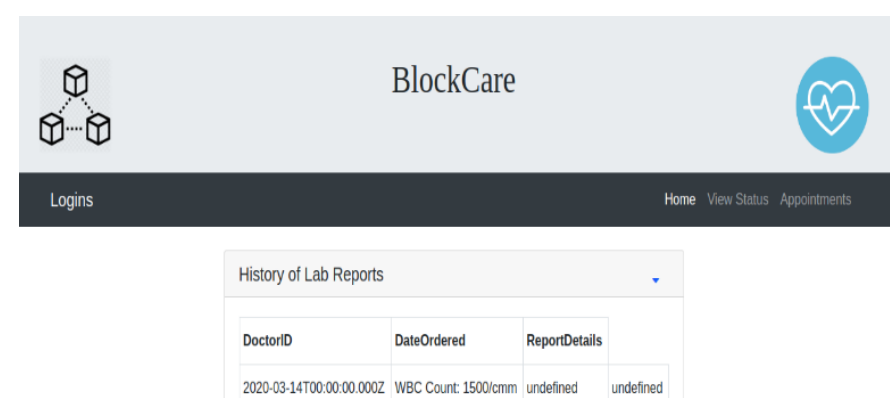

Fig. 10 Lab Report

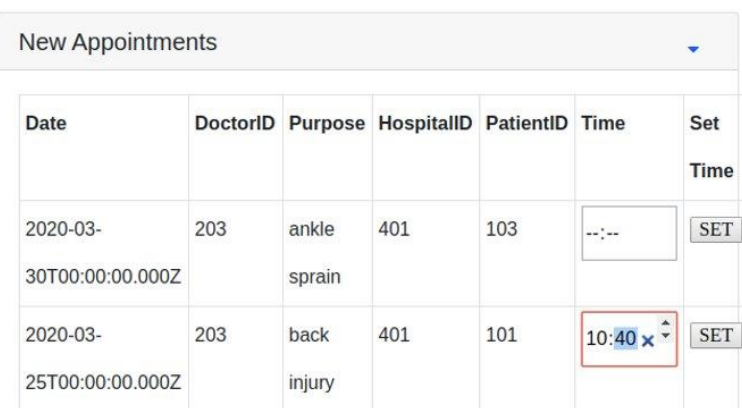

Fig. 11 Schedule Appointment

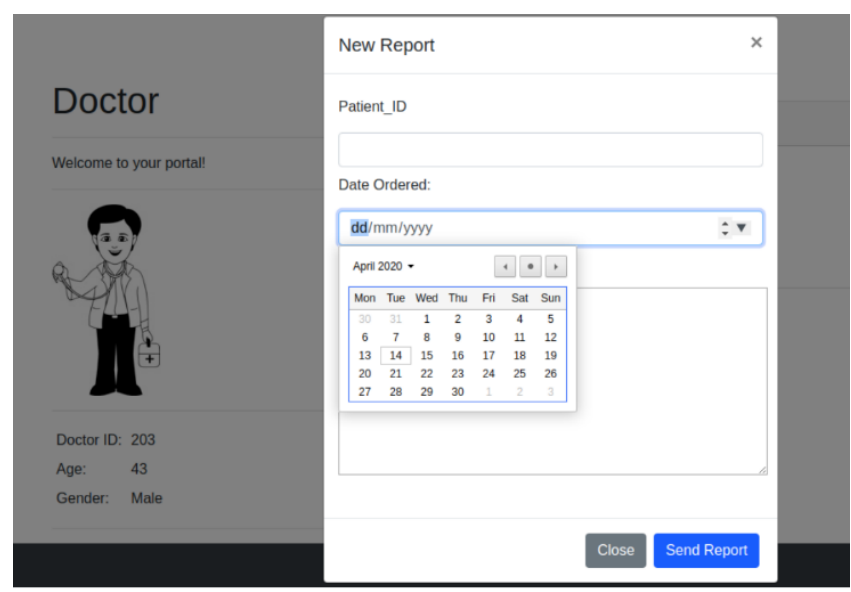

Fig. 12 Writing Prescription

ISSN: 2582-1369 (online)

Submitted: 18.11 .2020

Accepted: 08.12.2020

Published: 14.12.2020 
Journal of ISMAC (2020)

Vol.02/ No.04

Pages: 222-231

http://irojournals.com/iroismac/

DOI: https://doi.org/10.36548/jismac.2020.4.006

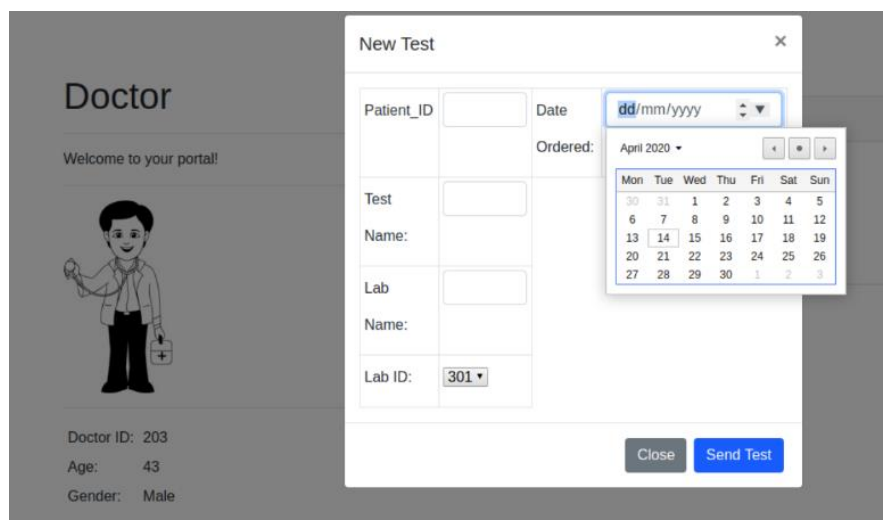

Fig. 13 Request Lab Report

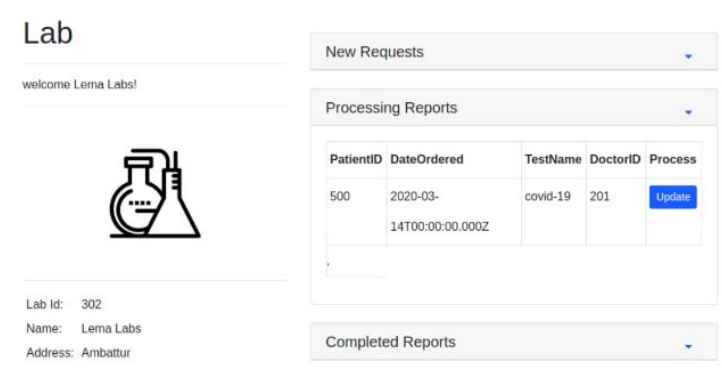

Fig. 14 Enter Lab Results

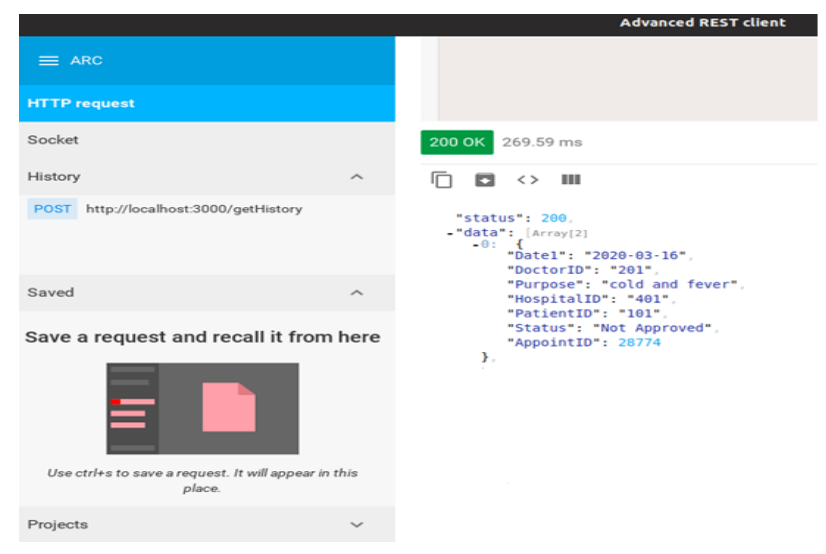

Fig. 17 Genesis Block 


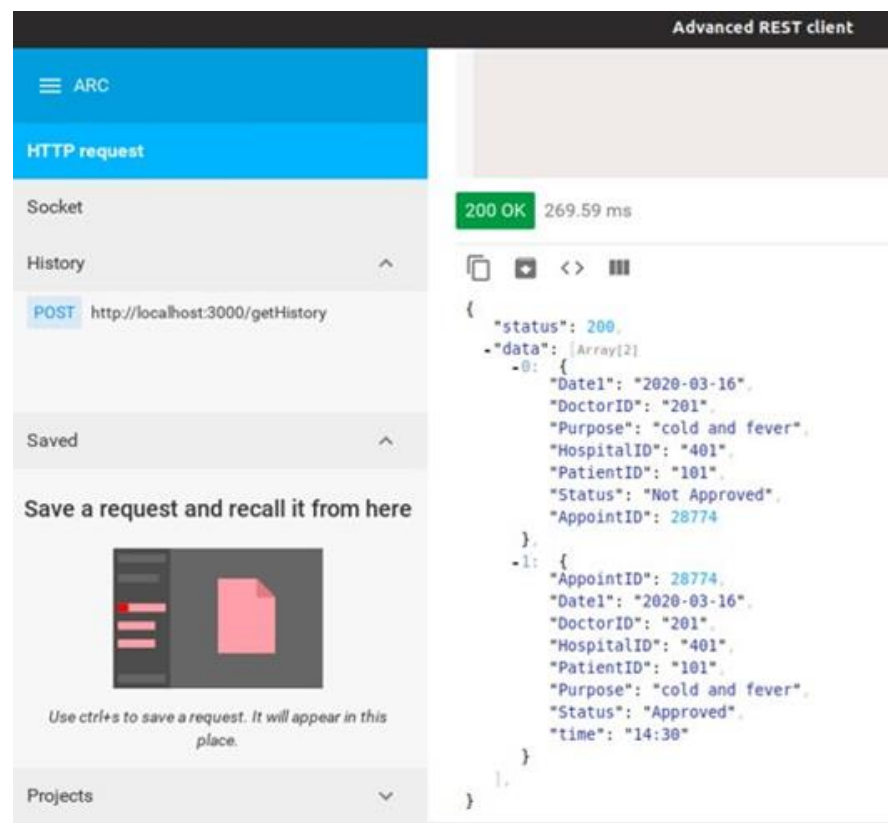

Fig. 18 Addition of the second block

\section{FUTURE WORK}

Our current application involves the use of Blockchain for a single hospital. This can further be scaled to include a larger sector of the hospitals across the nation.

Considering the EHRs, the blockchain is utilized as the solution for managing any of the nation's premier medical institutions. For instance, a company located in London named Medicalchain applied blockchain technology to store the EHRs to investigate shared ledger actions. These have happened with an agreement over the Mayo Clinic and Medicalchain. Massachusetts General Hospital has employed MediBloc, which is a Korean based company, uses the EHR system to store and interchange the information along with blockchain technology.

\section{v. CONCLUSION}

The beginning to the progression of blockchain technology evolved through Bitcoin. This technology introduced use cases in several industries including healthcare. The main aim of this research is to recognize the usage of blockchain technology in healthcare. The application developed in this research is to satisfy the challenges and shortcomings of the use cases in healthcare applications based on blockchain technology. The various use cases in the field of healthcare such as drugs and pharmaceutical supply chain management, management of electronic medical records, remote patient monitoring, biomedical research and education, health data analytics, amongst others.

Most of the healthcare applications consider blockchain technology and develop prototypes on the basis of emerging blockchain paradigms which include off-chain storage, smart contracts, permission blockchain, and so on. In the future, there is a need to perform more investigation based on the characterization, evaluation, and understanding of blockchain technology in the applications of healthcare. The research can also be conducted in healthcare using blockchain technology to analyze the various challenges based on latency, interoperability, scalability, privacy, and security. 


\section{ACKNOWLEDGEMENT}

This Work was done in collaboration with Rapidqube Digital Solutions' support and guidance

\section{REFERENCES}

[1] Nakamoto, Satoshi. "Bitcoin: A peer-to-peer electronic cash system." (2008): 28.

[2] Islam, SM Riazul, et al. "The internet of things for health care: a comprehensive survey." IEEE Access 3 (2015): 678-708.

[3] Ekblaw, Ariel, et al. "A Case Study for Blockchain in Healthcare:"MedRec" prototype for electronic health records and medical research data." Proceedings of IEEE Open \& Big Data Conference. 2016.

[4] Peterson, Kevin, et al. "A Blockchain-Based Approach to Health Information Exchange Networks." (2016). [5] Zhang, Jie, Nian Xue, and Xin Huang. "A Secure System For Pervasive Social Network-Based Healthcare." IEEE Access 4 (2016): 9239-9250. R. Nicole, "Title of paper with only first word capitalized," J. Name Stand. Abbrev., in press.

[5] Shae, Zonyin, and Jeffrey JP Tsai. "On the Design of a Blockchain Platform for Clinical Trial and Precision Medicine." Distributed Computing Systems (ICDCS), 2017 IEEE 37th International Conference on. IEEE, 2017.

[6] Benchoufi M, Porcher R and Ravaud P. Blockchain protocols in clinical trials: Transparency and traceability of consent [version 1; referees: 1 approved, 1 not approved]. F1000Research 2017, 6:66 (doi: 10.12688/f1000research.10531.1)

[7] Zhang, Yin, et al. "Health-CPS: Healthcare cyber-physical system assisted by cloud and big data." IEEE Systems Journal 11.1 (2017): 88-95

[8] Zibin Zheng, Shaoan Xie, Hongning Dai, Xiangping Chen and Huaimin Wang. "An Overview of Blockchain Technology: Architecture, Consensus, and Future Trends".

[9] D. Kraft, "Difficulty control for blockchain-based consensus systems," Peer-to-Peer Networking and Applications, vol. 9, no. 2, pp. 397-413, 2016.

[10] G. Foroglou and A.-L. Tsilidou, "Further applications of the blockchain," 2015.

[11] M. Sharples and J. Domingue, "The blockchain and kudos: A distributed system for educational record, reputation and reward," in Proceedings of 11th European Conference on Technology Enhanced Learning (EC-TEL 2015), Lyon, France, 2015, pp. 490-496.

[12] NRI, "Survey on blockchain technologies and related services," Tech. Rep., 2015. [Online]. Available: http://www.meti.go.jp/english/press/ 2016/pdf/0531 01f.pdf

[13] A. Chepurnoy, M. Larangeira, and A. Ojiganov, "A prunable blockchain consensus protocol based on non-interactive proofs of past states retrievability," arXiv preprint arXiv:1603.07926, 2016.

[14] M. Vukolic, "The quest for scalable blockchain fabric: Proof-of-work ' vs. bft replication," in International Workshop on Open Problems in Network Security, Zurich, Switzerland, 2015, pp. 112-125.

[15] V. Buterin, "On public and private blockchains," 2015.[Online].Available: https://blog.ethereum.org/2015/08/07/on-public-and-private-blockchains/

[16] D. Johnson, A. Menezes, and S. Vanstone, "The elliptic curve digital signature algorithm (ecdsa)," International Journal of Information Security, vol. 1, no. 1, pp. 36-63, 2001.

[17] J. Barcelo, "User privacy in the public bitcoin blockchain," 2014.

[18] “Antshares digital assets for everyone," 2016. [Online]. Available: https://www.antshares.org

[19] F. Tschorsch and B. Scheuermann, "Bitcoin and beyond: A technical survey on decentralized digital currencies," IEEE Communications Surveys Tutorials, vol. 18, no. 3, pp. 2084-2123, 2016. 